\title{
TAGUNG
}

\section{Die Stabilität Europas nach der Krise - Eine interdisziplinäre Betrachtung}

\author{
Tanja Hennighausen*
}

Die erste deutsche ECSA-Konferenz für Nachwuchswissenschaftler fand vom 21. bis 23. Februar 2011 in Berlin statt. Diese gemeinsam vom Zentrum für Europäische Wirtschaftsforschung (ZEW) und dem Arbeitskreis Europäische Integration (AEI) organisierte Tagung befasste sich mit den Auswirkungen der Finanz-, Wirtschafts- und Schuldenkrise auf die Stabilität Europas. Da die Stabilität politischer und ökonomischer Systeme von einer Vielzahl von Faktoren abhängt, wurde im Rahmen dieser Veranstaltung ausdrücklich ein interdisziplinärer Ansatz verfolgt: Nachwuchswissenschaftler aus Rechts-, Politik- und Wirtschaftswissenschaften befassten sich gemeinsam mit vier etablierten Vertretern der beteiligten Disziplinen mit den Folgen der Krise für die Europäische Union sowie deren Stabilität und erörterten vor diesem Hintergrund notwendige Reformschritte.

Die Folgen der Finanzkrise für Europa und den (rechtlichen) Handlungsspielraum der Europäischen Union

Ingolf Pernice eröffnete die Tagung mit einem Vortrag über verfassungsrechtliche Aspekte von EU-Maßnahmen zur Sicherung der Finanzmarktstabilität. In den Mittelpunkt seiner Argumentation stellte er die rechtlichen Grundlagen der Europäischen Union und des Euro. Während die Eurozone zwar eine gemeinsame Geldpolitik verfolgt, verfügen die Mitgliedstaaten weiterhin über eine autonome Haushalts- und Fiskalpolitik. Die im Vertrag von Maastricht geforderte fiskalische Disziplin könne aufgrund schwacher Regeln auf

\section{Europe's Post-Crisis Stability - An Interdisciplinary Approach}

Mit Unterstützung der Europäischen Kommission, der Stiftung „Geld und Währung“ und des

Zentrums für Europäische Wirtschaftsforschung.

Berlin, 21.-23. Februar 2011

Constitutional Issues of the EU Measures for Financial Markets Stability

Ingolf PERNICE, Humboldt University Berlin

Financial Crisis I

Challenges of EU State Aid Law in Times of

Crisis

Roman GUSKI, University of Heidelberg

The Financial Crisis and the Baltic States:

Effects on Government Structure in the Context of the European Structural Funds

Sigita URDZE, Technische Universität Darmstadt

Macroeconomic Aspects of the Crisis Cyclical Variability of the Ukrainian Economy Maryana PYRCH, Lviv Polytechnic National University, Lviv

The Impact of External Shocks on the Eurozone: A Structural VAR model

Jean-Baptiste GOSSÉ, Center for Economics of the University of Paris North

Asymmetric International Risk Sharing in the Euro Area

Holger ZEMANEK, University of Leipzig

Empires and Crises: The European Union in Historical Perspective

Gary MARKS, VU University Amsterdam/University of North Carolina at Chapel Hill

* Tanja Hennighausen, Wissenschaftliche Mitarbeiterin am Zentrum für Europäische Wirtschaftsforschung (ZEW), Mannheim. 
multinationaler Ebene nicht durchgesetzt werden, was wiederum die Angriffspunkte an den Finanzmärkten erhöhe. Trotz nationaler Maßnahmen, die im Zuge der Finanzkrise durch die Mitgliedstaaten ergriffen wurden, habe sich ein gemeinsames Rettungsprogramm für die Mitgliedstaaten der Eurozone (Euro-Staaten) als unvermeidlich erwiesen. Dessen rechtliche Grundlage sei aber weiterhin umstritten. Dabei habe gerade die aktuelle Krise die Notwendigkeit einer weiteren rechtlichen Stärkung der Europäischen Union verdeutlicht. Zunächst beträfe dies die Schaffung rechtlicher Rahmenbedingungen für Maßnahmen der Europäischen Union zum Schutz des Euro, längerfristig forderte Pernice aber eine gemeinsame Wirtschafts- und Sozialpolitik der Eurozone. Denn nur auf diesem Wege könne das Ziel der Lissabon-Strategie beziehungsweise von deren Nachfolgerin der Europa 2020-Strategie realisiert werden, die Europäische Union zur wissensbasierten und wettbewerbsfähigen Ökonomie zu machen.

Roman Guski betrachtete die beihilferechtliche Grundlage staatlicher Rettungspakete für Banken. Diese Maßnahmen seien zwar angesichts der Finanzkrise notwendig geworden, stünden aber in einem Spannungsfeld zum geltenden EU-Recht. Grundsätzlich seien staatliche Interventionen nur dann rechtlich legitimiert, wenn sie den freien Markt fördern. Die Kommission habe einen gewissen diskretionären Entscheidungsspielraum (zum Beispiel bei ernsthaften wirtschaftlichen Schwierigkeiten) bei der Genehmigung von Beihilfen, dennoch mussten angesichts der Finanzkrise neue rechtliche Grundlagen geschaffen werden, die speziell auf Rettungspakete für Banken und Finanzinstitute anwendbar seien. Im Rahmen der neuen EU-Gesetze für Beihilfen sei so ein Instrument zur Wiederherstellung der Stabilität geschaffen worden, das gleichzeitig einen Kontrollmechanismus für Staatsinterventionen der Mitgliedstaaten darstelle und so Marktverzerrungen auf ein Minimum reduziere.

Auch Rastislav Funta stellte in seinem Vortrag auf die Konsequenzen der globalen Fi-

\section{Financial Crisis II}

Global Financial Crisis, Financial Instability, Future of the Financial System and the EU Law Rastislav FUNTA, Charles University Prague

The Financial Crisis and the EU: New Regulatory Clothes for the Emperors?

Kilian SENG, University of Konstanz

\section{Debt Crisis I}

Stability and Growth Pact: Evidence Marcin WOLSKI, Tilburg University

The Common Default Risk Premium of the Euro Area and the Stability Pact: The Empirical Relevance of Credible Fiscal Institutions in a Monetary Union

Roman GOLDBACH, Georg-August-University Göttingen

The Sovereign Debt Crisis and the Future of the Eurozone

Paul DE GRAUWE, Katholieke Universiteit Leuven

\section{Debt Crisis II}

How to Prevent a Greek Tragedy? On the Credibility of Constitutional Debt Limits

Karsten MAUSE, University of Bremen

Debt Sustainability in Selected Euro Area Countries. Empirical Evidence Estimating

Time-Varying Parameters

Bettina FINCKE, University of Bielefeld

\section{Crisis-Related Aspects}

Causes of Corruption in European Countries:

History, Law and Political Stability

Muhammad Tariq MAJEED, University of Glasgow

The Effect of Macroeconomic Shocks on a Small Open Economy with Financial Accelerator:

Lessons for Eastern Europe

Dilyana DIMOVA, University of Oxford

The Political Economy of European Integration - EURO's Burdensome Legacy

Werner ABELSHAUSER, University of Bielefeld

nanzkrise sowie der Instabilität der Finanzmärkte für die Zukunft des Finanzsystems und der EU-Gesetzgebung ab. Trotz zahlrei- 
cher struktureller Reformen, die angesichts der Krise notwendig geworden seien, erscheine die Zukunft des Euro weiterhin ungewiss. Zur Förderung der künftigen Entwicklung der Eurozone sei daher eine Vielzahl weiterer Maßnahmen nötig. Insbesondere zählten dazu eine weitere Integration sowie eine Verpflichtung der Euro-Staaten zur Budgetdisziplin, die Bereitschaft der Europäischen Zentralbank ihre Mitglieder bei fiskalischen Schwierigkeiten zu unterstützen, die Einbeziehung des Internationalen Währungsfonds (IWF) bei künftigen Finanzkrisen und schließlich weitere Reformen der Arbeitsund Produktmärkte.

Kilian Seng befasste sich mit der Frage, ob die Finanzkrise zur Etablierung effektiver Regeln zur Überprüfung der Budgetdisziplin in der Eurozone beigetragen hat. Bei der Betrachtung unterschiedlicher Reformvorschläge habe sich gezeigt, dass in vielfacher Hinsicht die Chancen zu grundlegenden Reformen im Rahmen des Stabilitäts- und Wachstumspakts ungenutzt geblieben seien. Eine Ausnahme stelle eine mögliche Neuregelung des Entscheidungsprozesses hinsichtlich etwaiger Sanktionen bei Verstößen gegen die Regeln des Stabilitäts- und Wachstumspakts dar. Bisher sei für die Einleitung eines Defizitverfahrens gegen einen Euro-Staat eine qualifizierte Mehrheit im Rat der Europäischen Union erforderlich. Aufgrund der hohen Verschuldung in der Mehrzahl der Mitgliedstaaten habe dies deren Durchsetzung erschwert. Ein Reformvorschlag beziehe sich daher auf eine „Umkehr der qualifizierten Mehrheit". Mit einer solchen Regelung müsse für die Ablehnung eines Defizitverfahrens eine qualifizierte Mehrheit im Rat erreicht werden, was die Einleitung eines Verfahrens erleichtern sollte.

Die Auswirkungen der Finanzkrise auf Tendenzen zur politischen Dezentralisierung in den Baltischen Staaten stellte Sigita Urdze dar. Traditionell sei das Baltikum zentralistisch organisiert und auch im Zuge der Aufnahme in die Europäische Union blieben grundlegende Dezentralisierungstendenzen aus. Neben historischen Gegebenheiten sei dies auch auf die Folgen der Finanzkrise zurückzuführen. Die Finanzkrise habe zwar erhebliche wirtschaftliche Folgen für das gesamte Baltikum, dennoch unterscheide sich die Lage der drei Staaten deutlich voneinander: Während Estland als Folge der restriktiven Budgetregeln einen Haushaltsüberschuss erwirtschaftet habe, sei die Haushaltslage in den beiden anderen Baltischen Staaten kritischer. Grundsätzlich könne in einer solchen Situation auf die EU-Strukturfonds zurückgegriffen werden, die ihrerseits die Regionalisierung vorantreiben. Deren Inanspruchnahme sei aber an die Fähigkeit zur nationalen Kofinanzierung geknüpft, die von den Baltischen Staaten (mit Ausnahme von Estland) in der Krise nicht mehr in vollem Umfang geleistet werden könnte. Daher würden sich vor allem Litauen und Lettland gegenwärtig mit fiskalischen Problemen konfrontiert sehen, die weitere Dezentralisierungsprozesse behinderten.

\section{Makroökonomische Aspekte der Wirtschafts-,} Finanz- und Schuldenkrise

Maryana Pyrch ging der Frage nach, weshalb die ukrainische Volkswirtschaft eine starke Anfälligkeit für Konjunkturschwankungen aufweist. Im Vergleich zu den osteuropäischen Mitgliedstaaten der Europäischen Union habe sich der Übergang zur Marktwirtschaft in der Ukraine langsamer vollzogen. Gleichzeitig weise die Wirtschaft dieses Staates eine hohe Volatilität auf, die unter anderem aus der hohen Abhängigkeit der Ukraine von der Weltwirtschaft resultiere. Neben auBenwirtschaftlichen Faktoren spiele auch das konsumgetriebene Wachstum - finanziert über ausländische Kredite und staatliche Sozialpolitik - der vergangenen Jahre eine wesentliche Rolle. Die Aufnahme von Auslandskrediten habe in der Finanzkrise zu einer Abwertung der Währung geführt, während sozialpolitisch motivierte Lohnerhöhungen die Wettbewerbssituation des Landes weiter geschwächt hätten. Daher solle die Politik öko- 
nomische Reformen zur Verbesserung der Wirtschaftsstruktur auf den Weg bringen, um so die Abhängigkeit des Landes von externen ökonomischen Faktoren zu verringern.

Jean-Baptiste Gossé befasste sich mit den Folgen von externen Schocks auf die EuroStaaten. Im Mittelpunkt seiner zeitreihenökonometrischen Analyse stand dabei die Frage, inwieweit die Staaten in unterschiedlicher Weise auf solche Schocks reagieren. Während Ölpreisschocks oder monetäre Schocks (Rückgang der weltweiten Zinssätze) weitgehend ähnliche ökonomische Konsequenzen in den Mitgliedstaaten hätten, wirkten sich die Finanzkrise und globale Leistungsbilanzungleichgewichte unterschiedlich aus. Eben diese unterschiedlichen Reaktionen auf externe Schocks könnten einen erheblichen Teil der Unterschiede im Wirtschaftswachstum oder der Leistungsbilanzen in der Eurozone erklären.

Schließlich stellte Holger Zemanek auf die Asymmetrie in der internationalen Risikoteilung innerhalb der Eurozone ab. Die Eurozone zeichne sich durch wirtschaftliche Heterogenität der Mitgliedstaaten aus. Aufgrund der gemeinsamen Geld- und Währungspolitik müssten wirtschaftliche Anpassungsprozesse über andere Kanäle erfolgen. Eine Möglichkeit, asymmetrische Schocks und unterschiedliche Konjunkturzyklen abzuschwächen, basiere auf einer internationalen Risikoteilung innerhalb der Währungsunion. Die Euro-Staaten scheinen aber in unterschiedlichem Umfang an der internationalen Risikoteilung zu partizipieren. Zwar reduziere ein hohes Auslandsvermögen eines Staates Konsumschwankungen, aber eine asymmetrische Verteilung von Auslandsvermögen beziehungsweise -verbindlichkeiten schwäche die Risikoteilung in der Eurozone deutlich ab. Daher sollten weitere Maßnahmen (zum Beispiel eine weitere Deregulierung der Arbeitsmärkte) zur Stärkung der Anpassungsprozesse ergriffen werden.

\section{Die Schuldenkrise und deren Folgen für die Stabilität des Euro}

Paul De Grauwe betrachtete die Verschuldungskrise einiger souveräner Staaten und deren Auswirkungen auf die Zukunft der Eurozone. Trotz Konsolidierungsbemühungen sei die Europäische Union und nicht die USA von einer akuten Schuldenkrise betroffen. ${ }^{1}$ Ursächlich dafür sei das Fehlen von Stabilisierungsmechanismen zum Ausgleich divergierender nationaler wirtschaftlicher Entwicklung. Während die Währungsunion in den USA in eine politische (und budgetäre) Union eingebettet sei, fehlten solche Mechanismen innerhalb der Europäischen Währungsunion. So sei auch eine automatische Solidarität mit verschuldeten Mitgliedstaaten in der Europäischen Union mit der Begründung eines Moral-Hazard-Problems auf Seiten der verschuldeten Staaten ausgeblieben. Die Fokussierung auf das Regelwerk des Stabilitäts- und Wachstumspakts anstelle der Etablierung eines Stabilisierungsmechanismus verglich De Grauwe mit einer Situation, in der zunächst die Brandstifter bestraft werden, bevor die Feuerwehr mit dem Löschen beginne. Gleichzeitig basiere die Schuldenkrise in vielen betroffenen Staaten (außer Griechenland) auf privater und nicht staatlicher Verschuldung, die wiederum nicht durch striktere Fiskalregeln bekämpft werden könne. Ein möglicher Reformvorschlag wäre die Einführung eines Europäischen Währungsfonds als Ersatz für eine Budgetunion. Außerdem solle die Einführung von Eurobonds vorangetrieben werden.

Marcin Wolski beurteilte die Wirksamkeit des Stabilitäts- und Wachstumspakts auf Basis der Konsolidierungsbemühungen der EuroStaaten. Wenngleich die Euro-Staaten gegen die Regeln des Stabilitäts- und Wachstumspakts verstoßen hätten, habe sich die durchschnittliche Budgetdisziplin seit dem Inkrafttreten des Pakts verbessert. Eine weitere

1 Anmerkung der Autorin: Zum Zeitpunkt der Konferenz (Februar 2011) wurde noch nicht von einer US-Schuldenkrise gesprochen. 
Analyse von 13 Mitgliedstaaten zeige, dass zwar elf dieser Staaten im Durchschnitt ein Haushaltsdefizit aufwiesen, dennoch alle betrachteten Staaten - mit Ausnahme von Griechenland - ihre Haushaltsdisziplin im Zeitablauf verbessert hätten. Wenngleich der Stabilitäts- und Wachstumspakt zu einer Erhöhung der Budgetdisziplin in den Mitgliedstaaten beigetragen habe, verdeutliche die Erfahrungen der Schuldenkrise, dass weiteres Verbesserungspotenzial bestehe.

Roman Goldbach befasste sich mit der Frage, ob die Eurozone mit einer allgemeinen Risikoprämie für Staatsanleihen konfrontiert wird und untersuchte vor diesem Hintergrund die Relevanz glaubwürdiger Finanzinstitutionen innerhalb einer Währungsunion. Die gegenwärtige Krise habe verdeutlicht, dass die internationalen Finanzmärkte zwar je nach Kreditwürdigkeit der Schuldnerstaaten unterschiedliche Risikoprämien fordern, sich aber die Mitglieder einer Währungsgemeinschaft diese Risikoaufschläge teilen würden. Insbesondere eine Schwächung der institutionellen Rahmenbedingungen (Stabilitäts- und Wachstumspakt) könne zu einem Verlust der Kreditwürdigkeit der gesamten Eurozone führen. Goldbachs Einschätzung zufolge bestätigt sich dies auch in den empirischen Analysen zur Relevanz von Institutionen zur Sicherung der Stabilität: Vor allem die Europäische Kommission erscheine als ein wesentlicher Akteur für die Finanzmärkte, auf deren Entscheidungen und Statements reagiert würde. Damit zeige sich, dass für die Wahrung der Stabilität der Eurozone zwar kurzfristige Maßnahmen zur Stützung der schwächsten Euro-Staaten nötig seien, aber vor allem glaubwürdige institutionelle Rahmenbedingungen zur fiskalischen Kontrolle durch die Europäische Union geschaffen werden müssten.

Karsten Mause befasste sich mit der Frage, ob in der Eurozone eine Schuldenbremse nach deutschem Vorbild implementiert werden sollte. Dazu schlug er ein Schema zur Überprüfung der Wirksamkeit von institutionali- sierten Schuldenbremsen vor. Neben der generellen Glaubwürdigkeit von Schuldengrenzen (zum Beispiel Existenz von Schlupflöchern), müsse dazu die Fähigkeit einer Gebietskörperschaft zur eigenständigen Lösung von fiskalischen Problemen betrachtet werden (zum Beispiel über Autonomie in der Besteuerung). Darüber hinaus solle die Möglichkeit der Anwendung von Insolvenzrecht auf eine Jurisdiktion betrachtet werden. Dann müsse die Wahrscheinlichkeit eines , bailouts' durch andere staatliche Ebenen oder durch die Europäische Zentralbank geprüft werden. Schließlich könnten auch Finanzmärkte als Disziplinierungsmechanismus gegen eine zu hohe Verschuldung wirken, da steigende Risikoprämien Konsolidierungsmaßnahmen erzwingen könnten.

Bettina Fincke stellte in ihrer quantitativen Untersuchung auf die Nachhaltigkeit der Verschuldung in ausgewählten Euro-Staaten ab. Dies sei vor allem vor dem Hintergrund der Regeln des Stabilitäts- und Wachstumspakts relevant, da diese eine Anhäufung von Schulden durch die Mitgliedstaaten der Europäischen Währungsunion unterbinden sollen. Auf Basis einer empirischen Analyse könne gezeigt werden, dass Frankreich und Deutschland sowie in der Tendenz auch Irland, Portugal und Spanien eine nachhaltige Verschuldungspolitik aufweisen. Lediglich Griechenland weise einen öffentlichen Schuldenstand auf, der nicht als nachhaltig gelte.

\section{Weitere Aspekte der politischen und ökonomi-} schen Stabilität in Europa

Die Ursachen von Korruption in europäischen Staaten betrachtete Muhammad Tariq Majeed in seinem Vortrag. Dabei sei Korruption kein Phänomen, das ausschließlich in Entwicklungsländern zu beobachten ist. Auf Basis international vergleichender Zeitreihen zeige sich ein Anstieg der Korruption in Europa, was in deutlichem Gegensatz zur allgemein vertretenen Meinung stehe, dass Korruption mit zunehmender wirtschaftlicher Entwicklung zurückgehe. In Zusammenhang mit dem 
Auftreten von Korruption habe sich vor allem die Gesetzgebung, politische Stabilität und die Geschichte eines Staates als relevant erwiesen: Es zeige sich, dass eine ausgeprägte Rechtsstaatlichkeit ebenso wie politische Stabilität mit einem geringeren Ausmaß an Korruption einhergehe.

Dilyana Dimova befasste sich mit den Auswirkungen ökonomischer Schocks im Zuge des Transformationsprozesses und der Finanzmarktliberalisierung in zehn osteuropäischen EU-Mitgliedstaaten. Die Aussagen basierten auf einem theoretischen Modell, in dem sich die privaten Haushalte in ihrer Fähigkeit Kredite aufzunehmen unterscheiden. Dimova untersuchte die Auswirkungen von wirtschaftlichen Schocks unter Berücksichtigung dieser unvollkommenen Kapitalmärkte, wie sie in Osteuropa zu beobachten seien. Dabei zeige sich, dass diese Marktunvollkommenheit die Wirkung von positiven Schocks (in Form von Produktivitätsfortschritten, besserem Zugang zu Krediten für private Haushalte, sinkenden Importpreisen und fallenden Risikoaufschlägen) auf die Produktion und den Konsum verstärken könne.

Europäische Integration aus historischer Perspektive

Gary Marks stellte die Finanzkrise zunächst in einen historischen Kontext und betrachtete verschiedene Weltreiche und deren Krisen. Dazu kontrastierte er die Europäische Union mit anderen europäischen Reichen innerhalb der letzten zwei Jahrtausende, da die europäische Integration vor dem Hintergrund eines langen Prozesses, der seine Anfänge im Römischen Reich hatte, betrachtet werden solle. Ein vorherrschendes Motiv für die Gründung und Ausweitung von Reichen sei die Ausnutzung von Größenvorteilen in der Bereitstellung öffentlicher Güter. Gleichzeitig erschwere das Zugehörigkeitsgefühl der Menschen zu kleineren Gemeinschaften die Schaffung größerer Staatsgebilde. Der Bestand und die Weiterentwicklung von Weltreichen hingen zudem wesentlich von deren
Fähigkeit zur Erhebung eigener Steuern ab. Dass der Haushalt der Europäischen Union bislang noch sehr gering sei (circa 1 Prozent des Bruttoinlandsprodukts der Europäischen Union), lasse sich auch dadurch erklären, dass die Europäische Union lediglich ein loser Zusammenschluss von Staaten sei. Um ein Reich oder einen Staatenbund zu stärken und dauerhaft erhalten zu können, sei dessen Akzeptanz durch die Bevölkerung von wesentlicher Bedeutung. In der Europäischen Union sei diese nur in sehr abgeschwächter Form gegeben (circa 10 Prozent der Bürger bezeichneten sich selbst als Europäer). In den empirischen Daten fällt allerdings der Unterschied in der Meinung von Eliten und der öffentlichen Wahrnehmung bezüglich des Nutzens der Europäischen Union für die eigene Nation auf. Gerade die allgemeine Skepsis innerhalb der Bevölkerung schränke die politischen Möglichkeiten einer weiteren Integration ein.

Werner Abelshauser befasste sich in seiner Rede mit der Geschichte des europäischen Integrationsprozesses. Der Grundstein dazu sei bereits nach dem zweiten Weltkrieg mit dem Marshall-Plan der USA und der damit verbundenen Gründung der Organisation für europäische wirtschaftliche Zusammenarbeit (OEEC) gelegt worden. Auch sei bereits im Jahr 1952 eine gemeinsame europäische Währung angedacht gewesen. Betrachtet man die Montanunion als Startpunkt der wirtschaftlichen Integration Europas, so hätten damals bei den Hauptakteuren, Deutschland und Frankreich, politische Motive dominiert: Während Frankreich seinen Nachbar durch die europäische Integration besser zu kontrollieren glaubte, habe die Bundesrepublik auf diesem Wege seine Souveränität wiedererlangen wollen. Eine supranationale Regierung sei für beide Zwecke weder notwendig noch angedacht gewesen, der Integrationsprozess sollte vielmehr langsam vorangetrieben werden. Die Geschichte der europäischen Integration zeige also, dass nicht wirtschaftliche, sondern politische Motive deren Triebfeder waren und dass die Abschaffung nationaler Souveränität nie geplant gewesen sei. Damit 
könne die Eurozone auch aus der aktuellen Krise nicht durch die Schwächung nationaler Souveränität ihrer Mitgliedstaaten gerettet werden, vielmehr werde die Europäische Union weiterhin eine Vertragsgemeinschaft souveräner Staaten bleiben. Angesichts der gemeinsamen Währung wäre eine Transferunion zwar notwendig, aber politisch eher nicht durchsetzbar.

\section{Staatlichkeit und Governance in Transformation}

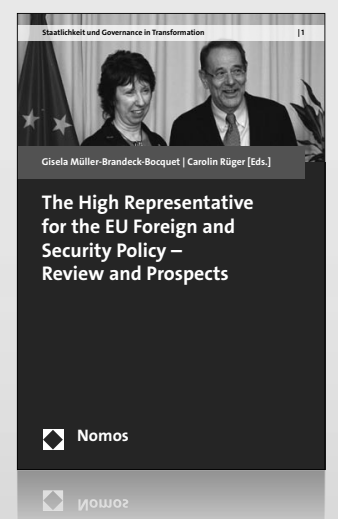

The High Representative for the EU Foreign and Security Policy Review and Prospects

Herausgegeben von Prof. Dr. Gisela Müller-Brandeck-Bocquet | Carolin Rüger 2011, 303 S., brosch., 54,-€ ISBN 978-3-8329-6002-5

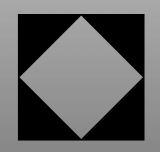

